\title{
A Review of Surgical Rehabilitation of the Upper Limb in Quadriplegia
}

\author{
B.R. Johnstone, MBBS, ${ }^{1}$ C.J. Jordan, B App Sc $(\mathrm{OT}){ }^{2}$ J.A. Buntine, \\ FRACS $^{3}$ \\ ${ }^{1}$ Former Surgical Registrar, ${ }^{2}$ Occupational Therapist, ${ }^{3}$ Former Senior Plastic \\ Surgeon, Spinal Injuries Unit, Austin Hospital, Heidelberg, 3084 Victoria, \\ Australia.
}

\section{Summary}

The options for surgical reconstruction of the quadriplegic upper limb are clarified by a new international classification of each limb independently, based upon the lowest functioning key muscle and residual sensation. Surgical restoration of active elbow extension, of pinch, and of grasp is now an accepted part of rehabilitation. This additional function may be achieved by transfer of a non-essential muscle, by tenodesis, or occasionally by arthrodesis. The techniques available for each group of the new international classification are described.

Key words: Quadriplegia; Rehabilitation; Hand surgery; Tendon transfer; Tenodesis.

Patients suffering from the devastating effects of quadriplegia may benefit from transfer of selected active muscles directly into the tendons of paralysed key muscles or indirectly, for example, transfer into a wrist extensor thus enhancing natural tenodesis (the tendency of the fingers to flex as the wrist extends). Surgical rehabilitation must be tailored to a patient's specific needs, personality and neurological state. Well planned muscle transfers may greatly help a very disabled person but these largely subjective gains are difficult to quantify. As Sterling Bunnell very aptly said 'if you have nothing, a little is a lot'. Hentz et al., (1983) found that a patient's assessment of functional results is more reliable than complex objective evaluations.

Bunnell (1948) suggested flexor tenodeses for quadriplegic patients. Many complex procedures, particularly those involving multiple arthrodeses, led to clawed, inflexible and unattractive hands. Muscle transfers established for other forms of paralysis tended to fail when applied to quadriplegia, but a number of reliable procedures have been developed in recent years to address the unique functional requirements of quadriplegia. The weight bearing function of the upper limbs must be maintained, particularly for pressure care and for transfers 
Table I International classification of the upper limb in quadriplegia (Giens, 1984)

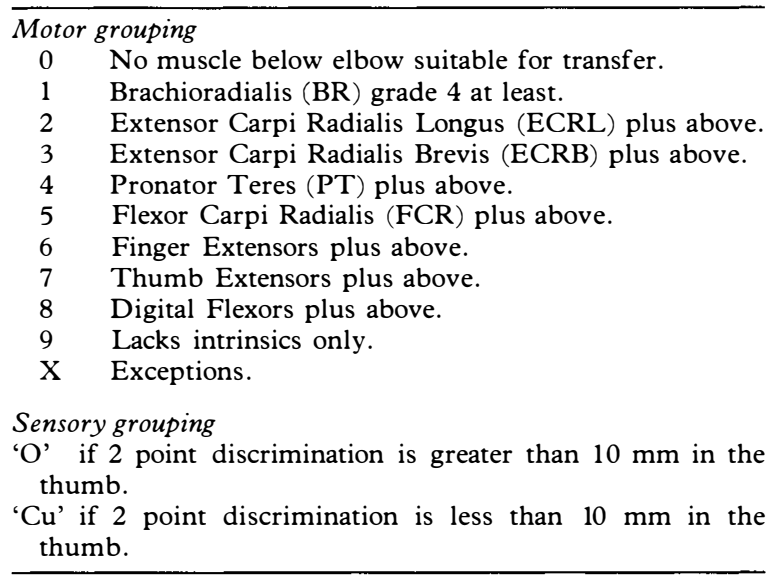

to and from wheelchairs, etc. Softness and flexibility are important for interpersonal contact and so must also be maintained. Moberg (1975), House et al. (1976), Freehafer et al. (1974), Lamb and Chan (1983) and others have reported encouraging results. Zancolli (1975) studied 97 complete quadriplegics in Buenos Aires between 1949 and 1974 . The largest group $\left(74_{\circ}^{\circ}\right)$ had active wrist extension ('complete quadriplegia below C6') and so surgery could provide lateral pinch and grip (Groups $2 \mathrm{~A}$ and 2B, by Zancolli's classification, see Table III). The results were better in patients with more complete function of the C6 cord segment, that is, strong wrist extension plus some activity of pronator teres (Group 2B II), flexor carpi radialis (Group 2B III) and triceps. Reconstructive procedures were performed on 76 of the 97 patients. Moberg (1978) believed that at least $60^{\circ}$ of quadriplegics could benefit from surgery.

Before surgery is planned, careful assessment of the residual power of each muscle of each arm is essential. As function is commonly asymmetrical, a classification by neurological level such as C6 or C7 is inadequate. Cutaneous sensibility, specifically two point discrimination in the thumb pulp, influences the effectiveness of restored pinch grip. There is often a disparity between the motor and sensory levels. If proprioception is inadequate, bilaterally reconstructed hands cannot be used independently as vision alone controls movement.

Satisfactory results require meticulous surgical technique, a lengthy period of postoperative immobilisation, and a demanding rehabilitation programme. As any independence achieved preoperatively is temporarily lost, the patient must be highly motivated. The team of medical and paramedical staff must have a special interest in this area and provide continuity of care.

\section{International Classification}

In October 1984, the Spinal Cord Injury Committee of the International Federation of Societies for Surgery of the Hand meeting in Giens, France, (McDowell et al., 1986), modified an International Classification, similar to that developed by Moberg (Table I). When only occular afferent impulses are avail- 
Table II Modified myotome table from Zancolli (1975). Comparison of various classification systems with neurological levels and the lowest functionmg muscies, Diagrams show the distribution of the dermatomes

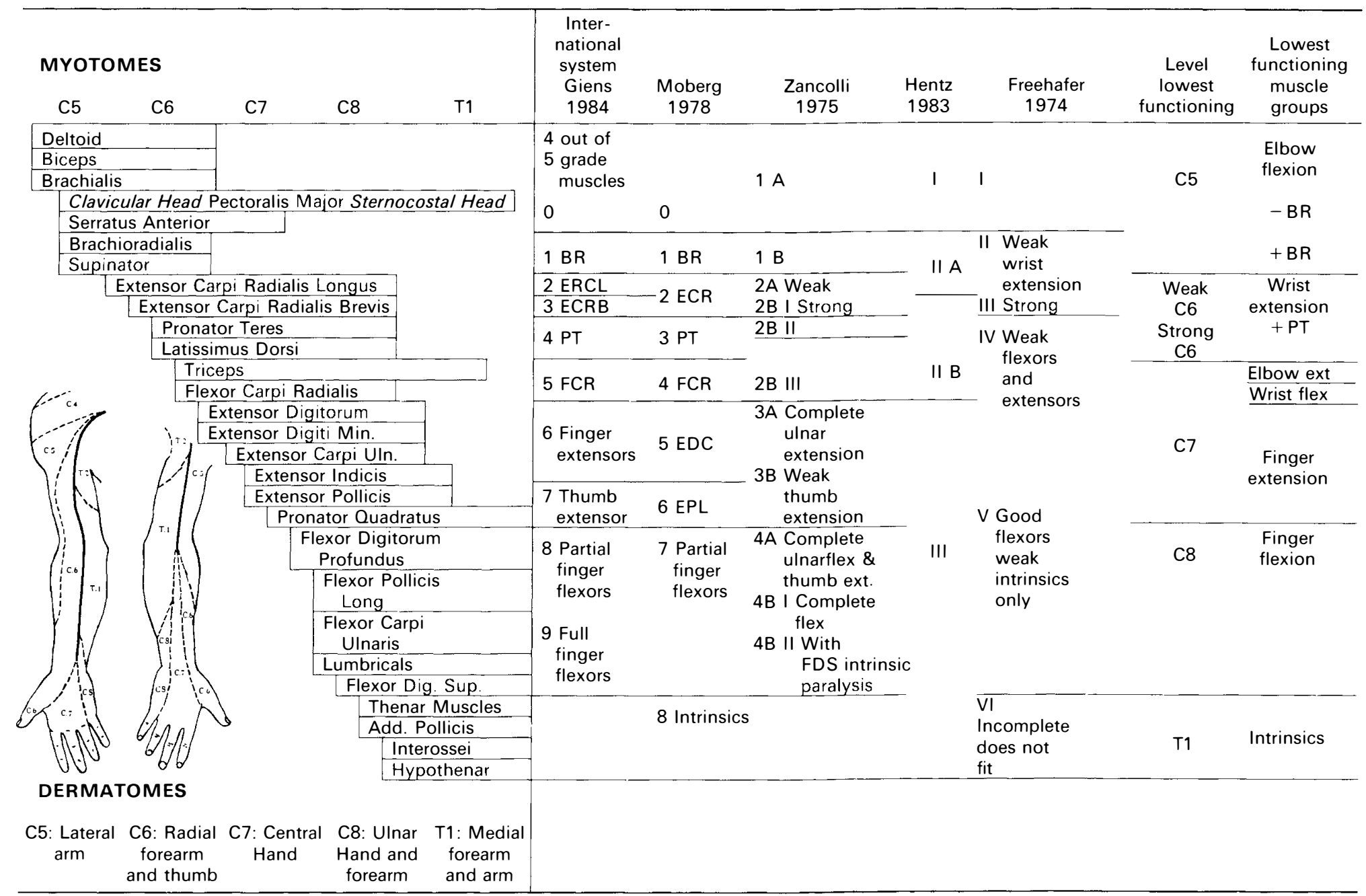


Table III This table compares the International Classification with the previously described classifications of Moberg, Zancolli, and Hentz. The relative frequencies (in percentages) of limbs belonging to each group of the Moberg, Zancolli and Hentz classifications, as published by the respective authors

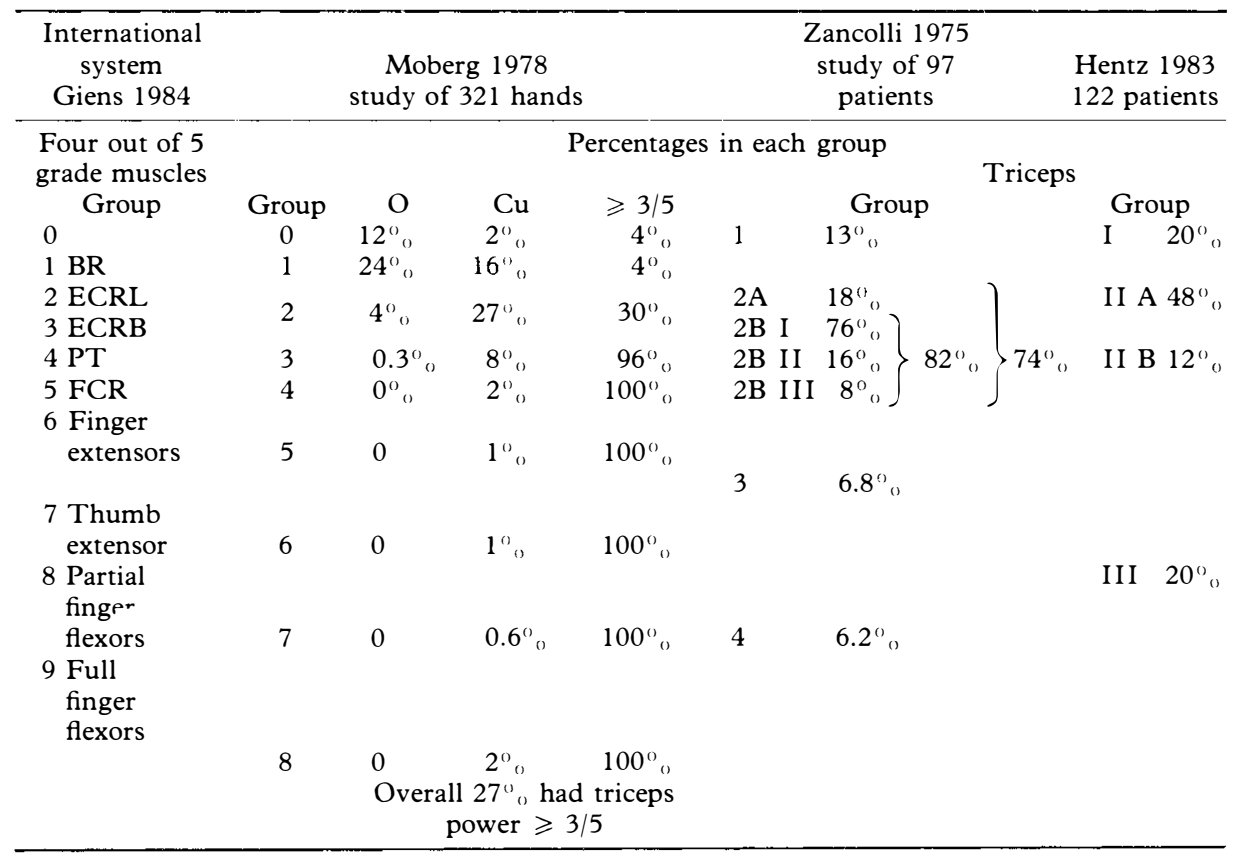

able, sensibility is designated 0 . If cutaneous two point discrimination is $10 \mathrm{~mm}$ or better in the thumb, sensibility is designated $\mathrm{Cu}$.

The motor component relates to muscles below the elbow which have adequate strength for transfer. This is grade 4 out of 5 (against gravity plus some resistance) on the Medical Research Council Scale (1954). Another key muscle is added for each group (the lowest adequately functioning muscle appearing on a standard myotome table, see Table II). For example, $\mathrm{Cu} 2$ denotes adequate strength in brachioradialis and extensor carpi radialis longus with sensation preserved in the thumb. Triceps function is classified separately.

Brachioradialis (BR) is tested indirectly by assessing tone during forced elbow flexion against resistance at $90^{\circ}$. In practice, this muscle performs weakly if transferred distally unless elbow flexion is opposed by a functional triceps or by a triceps tendon activated by a posterior deltoid or a biceps transfer. As the level of innervation of extensor carpi radialis brevis (ECRB) is slightly lower than that of extensor carpi radialis longus (ECRL), ECRB must be shown to be capable of independently achieving strong wrist extension before ECRL is transferred. Strong contraction of the muscle belly of ECRB may be palpable or its tendon may be felt distally as an independent rigid structure. Alternatively, function of pronator teres or flexor carpi radialis suggests that ECRB will be of adequate strength.

However, if there is still doubt, ECRB should be assessed separately from ECRL at operation by fixing to its tendon a weight via a pulley system, after 
exposure under local anaesthetic. It is considered capable of independent wrist extension if $5 \mathrm{~kg}$ can be lifted.

Table II is a myotome table showing the group number of the International Classification beside the lowest useful muscle of each group. Other classifications are shown for comparison, as is the neurological level and a dermatome diagram. The results of surveys by Zancolli (1975), Moberg (1978) and Hentz et al. (1983), showing the percentage of patients belonging to each group according to their own classification system, are shown in Table III.

\section{General principles of tendon transfer in quadriplegia}

Moberg (1978) described softening, stretching and contracture of collateral ligaments and volar plates over many years, due to the unbalanced tone of paralysed muscles, the effects of gravity and the absence of proprioception. However, the volar ligament of the thumb carpometacarpal joint and the collateral ligaments of the metacarpophalangeal joints of the fingers remain reliable. The quadriplegic loses voluntary control over most or all of the 37 muscles acting on the wrist and hand. When a transfer procedure is performed, a number of these muscles are replaced by one or two possibly weakened muscles. A relatively strong transferred muscle may cause a contracture over a long period, particularly if its new action is inadequately antagonized (Hentz et al., 1983).

Tendon transfers should not usually be considered until patients have been neurologically stable for at least 6 months. Patients need time to accept their disability, to maximise strength in their remaining muscles and to develop new skills.

Marked spasticity, particularly if it causes deformity, is a contraindication to surgery. This is more often a feature of incomplete lesions. Milder spasticity may be improved by selective division of specific nerves or tendons after trial nerve blocks. Other causes of generalised spasms should be excluded, such as pressure sores or urinary calculi. Hypersensitivity and painful paraesthesiae are also contraindications. Contractures should be prevented or, if present, treated actively except in cases where they have an adaptive role.

Other co-existing problems, such as pressure sores, should be treated before hand as enforced periods of bed rest may interfere with later rehabilitation which, like assessment, should be conducted with the patient in a chair. Suitably motivated patients seldom develop severe pressure sores. Neither age nor the duration of quadriplegia are contraindications, but the results of surgery are said to be less predictable if performed over 5 years after injury (Hentz et al., 1983). Poor motivation and psychological instability adversely affect the outcome.

Well established principles of tendon transfer surgery should be upheld. Namely:

1. The strength of the muscle to be transferred should be adequate to perform the desired function and to overcome any mechanical disadvantage including post-operative adhesions.

2. Ideally the motor should be a synergist of the movement to be restored, e.g. wrist extensors transferred to finger flexors. 
3. The muscle should be expendable. It is now accepted that an active flexor carpi radialis (C7 mainly) should not be transferred, as active wrist flexion is important for weight bearing, wheelchair propulsion and for powering extensor tenodeses to achieve thumb and finger extension. ECRL should not be transferred if ECRB is weak.

4. The joints to be activated should have an adequate range of passive movement.

5. The excursions of the transferred muscle and the recipient tendon should be comparable. The $3 \mathrm{~cm}$ excursion of the triceps tendon should be matched by freeing the attachments of the posterior part of the deltoid muscle prior to transfer. Brachioradialis must also be extensively freed to achieve a reasonable excursion. If a tendon crosses the wrist joint, its amplitude is increased by 2 to $3 \mathrm{~cm}$. through its full range by movement at this joint.

6. The line of action should be as straight as possible. If the tunnel in which a transferred tendon is placed is not straight, the tendon will effectively lengthen with time as the tunnel assumes a more direct line.

7. It was customary to perform only reversible procedures but, as the risk of functional loss is now negligible, this consideration is of less importance.

8. If a transferred muscle is to perform effectively a secondary action, there must be an effective antagonist to its primary action e.g. transferring brachioradialis distally is more effective if the triceps is strong or if active elbow extension is provided by a transfer of the posterior part of the deltoid muscle.

9. Surgery should be atraumatic with minimal dissection so as to decrease the formation of adhesions.

10. The transfer should be tested intra-operatively. This may be done using electrical stimulation during general anaesthesia (Freehafer et al., 1979), alternatively anaesthesia may be satisfactory after infiltrating the operative site with bupivacaine. It should be noted that when using a tourniquet, temporary paralysis occurs after 20 minutes of ischaemia and may take up to 20 minutes to recover.

Wrist extension is fundamental to restoration of grasp and pinch in quadriplegia, either by construction of a tenodesis or as a synergist of powered flexors. If extension of the wrist is weak, the tendon of the brachioradialis may be transferred into that of extensor carpi radialis brevis (ECRB), which has the most central line of action. If the ECRB has some power, an end to side anastomosis is performed but, if ECRB is quite weak, it is partially excised and an end to end anastomosis is performed by weaving to lessen the risk of post operative adhesions (Moberg, 1975). The pronated hand opens as gravity passively flexes the wrist (natural tenodesis). For most quadriplegic patients fusion or tenodesis of the wrist should be avoided, one exception being fusion in conjunction with the winch procedure (McDowell et al., 1986).

Surgical rehabilitation may best commence with provision of active extension of the elbow. The standard Moberg posterior deltoid transfer requires 6 weeks of immobilisation of the elbow in extension and another 3 months of strictly controlled mobilisation and rehabilitation. This procedure is very useful in its 
own right and also improves the effectiveness of a transferred brachioradialis. If grip reconstruction is performed first, hand retraining and independence are interrupted for a number of months if an elbow extension procedure is then performed.

Lateral pinch or key grip is the desired goal except for those patients who lack merely intrinsic function. (International Classification Groups 8 and 9). Two or three point precision grip can be achieved in these less disabled patients but the key grip, used for $50^{\circ}$ o of our gripping actions, is stronger, provides a broader gripping surface, is preferable cosmetically and is easier to achieve (Moberg, 1975).

The dominant hand should be treated first unless the non-dominant hand has significantly greater sparing of power or sensibility. Alternatively, the patient may request a trial procedure on the less important hand.

\section{Pre-operative programme}

At initial assessment, we (Johnstone et al., 1987) note the history of spinal cord and associated injuries, employment and social circumstances and levels of independence. Goals, expectations and motivation are carefully evaluated. Assessment of upper limb function includes documentation of residual sensation, the strength of all muscles, ranges of movement and the use of assistive devices. The patient is informed concerning the specific surgery planned and the gains which may realistically be expected. Possible complications, increased dependency in the post-operative period, and appropriate coping strategies are discussed. Candidates for surgery are encouraged to meet previously treated patients. It is stressed that surgery will not produce a normal hand and that maximum benefits depend upon a high level of personal commitment and motivation.

Stretching and muscle strengthening activities, sometimes combined with biofeedback, facilitate the isolated contraction of muscles to be transferred. In some patients, modification of previously learned techniques such as transfers, pressure lifts and wheelchair handling is required to prevent over stressing of tendon transfers or to accommodate altered wrist or finger posture. Special splints such as an adjustable hinge splint for deltoid to triceps transfer, an electric wheelchair for the rehabilitation phase and subsequent attendant care are arranged prior to surgery (Fig. 1).

\section{Post-operative programme}

Splints are applied post-operatively for a variable period depending on the procedure. Hands are often immobilised for 3 to 4 weeks. Post operative care includes control of oedema, and movement of uninvolved joints. At a later date, graded active exercise of transferred muscles is carefully introduced followed by graded upper limb activities.

Biofeedback increases motivation during therapy and may assist motor reeducation by teaching the patient to perform isolated contraction of transferred muscles. Transferred muscles are protected from undue strain, such as would result from independent pressure lifts, for a 3 month period. Static or dynamic 


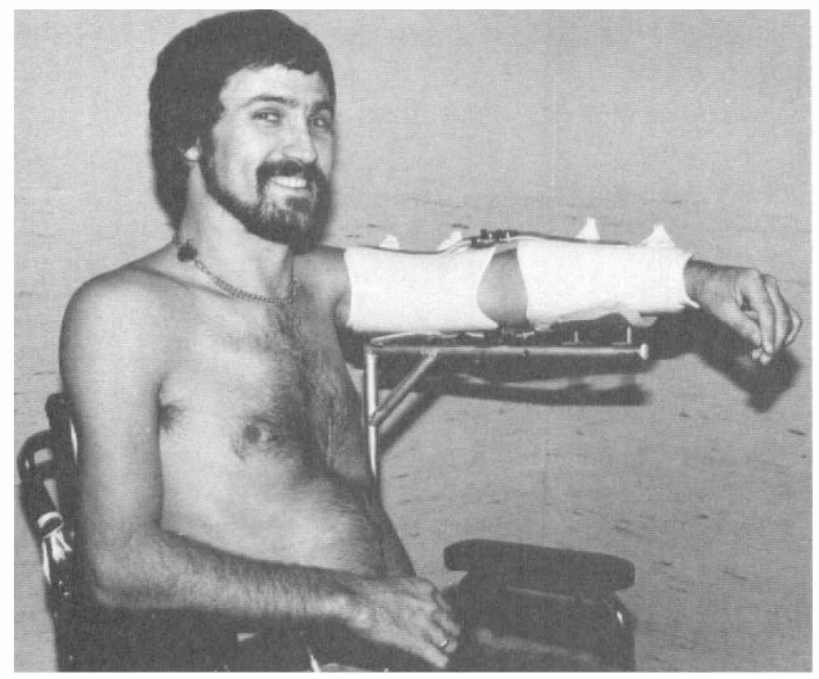

Figure 1 Adjustable elbow hinge splint and pivoting arm support attached to a electric wheelchair for the post-operative phase of a posterior deltoid to triceps transfer.

splints which may involve traction by elastic bands may be helpful. Night splinting helps to prevent contractures and may influence the tightness of a tenodesis.

\section{Procedures used in C5 quadriplegia: International classification group $\mathbf{O}$ [No muscle below the elbow suitable for transfer]}

These patients have no hand function and lack active wrist extension which cannot easily be corrected due to paralysis of the brachioradialis.

Moberg (1975) has transferred brachialis to ECRB using a tendon graft to achieve some active wrist extension. In this group of patients, fusion of the wrist after a trial of Kirschner wire stabilisation may be helpful as these patients usually require an electric wheelchair and are incapable of independent transfers. Wrist fusion may simplify the type of splint or orthosis used, alternatively the wrist may be held in an extended position by tenodesis of the ECRB. Provision of active extension of the elbow is possible and quite helpful (Hentz et al., 1983 and Freehafer et al., 1984).

Brummer (McDowell et al., 1986) has described a winch procedure where the tendon of flexor pollicis longus (FPL) is rerouted around the ulna and attached to it dorsally. As the radius is supinated by biceps, the tendon of FPL tightens pulling the thumb into flexion. The annular ligament is divided to increase the mechanical advantage of the tendon and the interphalangeal and wrist joints are stabilized (Fig. 2). Up to $2.5 \mathrm{Kg}$ can be applied to a $2 \mathrm{~cm}$. object and up to 5.8 $\mathrm{Kg}$ to a $4 \mathrm{~cm}$. object.

Buntine (Johnstone et al., 1987) has advanced the clavicular attachment of the anterior third of the deltoid muscle medially to compensate for absent pectoralis major function in a high quadriplegic (Fig. 3). As the ipsilateral biceps was the only other active upper limb muscle, improved control at the shoulder allowed him to bring his splinted hand to his mouth. 


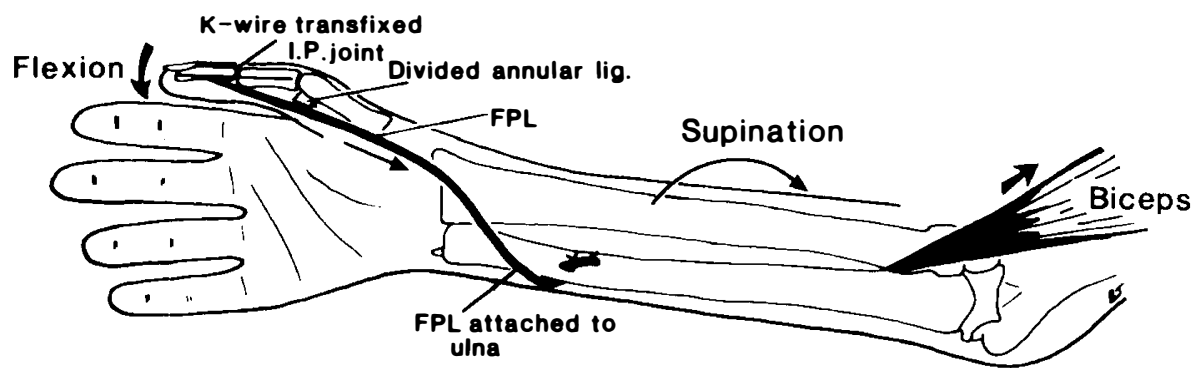

Figure 2 Brummer's Winch procedure: the re-routed flexor pollicis longus (FPL) tightens as the radius is supinated by the active biceps muscle.

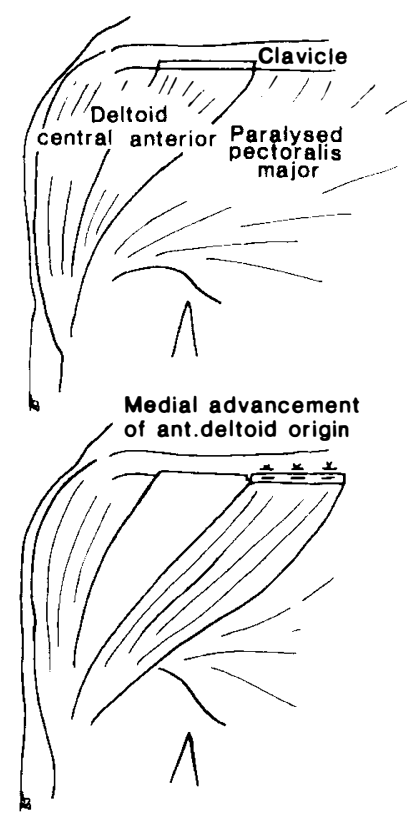

Figure 3 The bony origin of the anterior third of the deltoid is advanced medially to compensate for paralysis of the pectoralis major.

\section{Procedures used in C6 quadriplegia: International classification groups $1,2,3$ and 4}

Group 1) Brachioradialis $[B R]$ only Functioning

Group 2) Extensor Carpi Radialis Longus [ECRL] Functioning

Group 3) Extensor Carpi Radialis Brevis [ECRB] Functioning

Group 4) Pronator Teres [PT] Functioning

\section{Moberg pattern}

Moberg's key grip procedure consists of tenodesis of the flexor pollicis longus (FPL) tendon to the volar surface of the radius and stabilisation of the thumb interphalangeal (IP) joint with a buried Kirschner wire (Moberg, 1975). Weak active wrist extension may be augmented by transfer of brachioradialis into 


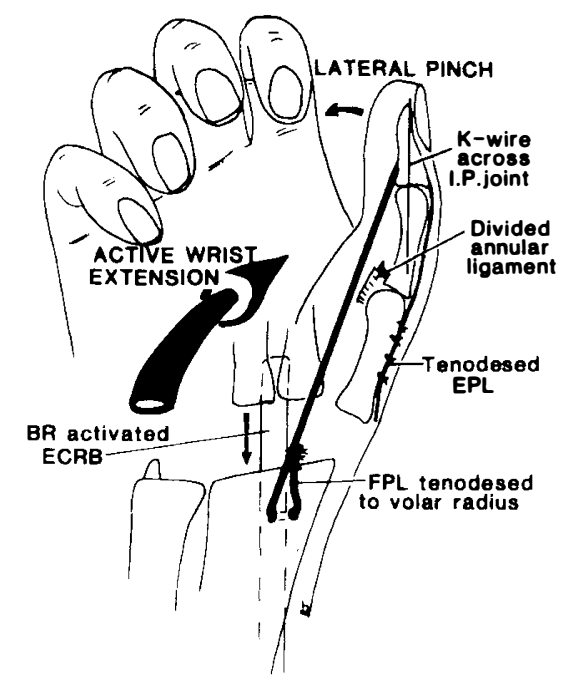

Figure 4 Moberg's key grip procedure: Flexor pollicis longus (FPL) tenodesed to volar radius, extensor pollicis longus (EPL) tenodesed to first metacarpal, Kirschner wire interphalangeal (IP) joint stabilization, divided annular ligament (now abandoned) and wrist-extensor tendon (ECRB) activated by branchioradialis (BR). Active wrist extension tightens the FPL tenodesis causing pinch of the thumb against the lateral aspect of the index finger.

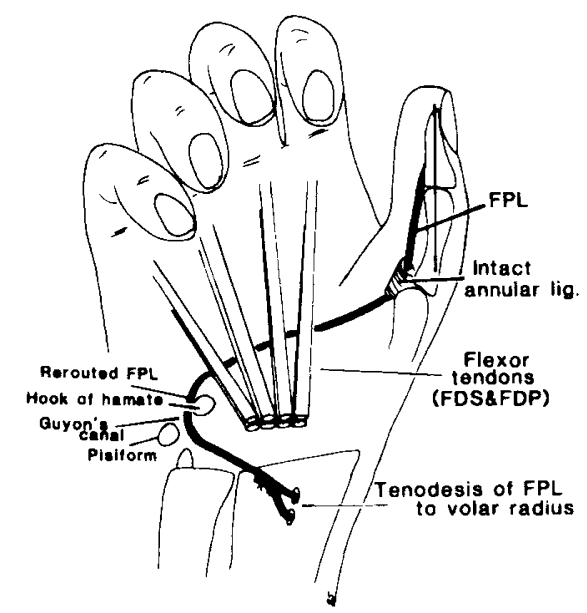

Figure 5 Brand's modification of Moberg's key grip: FPL passes from the intact annular ligament, deep to the flexor tendons, through Guyon's canal (between hook of the hamate and pisiform) to the volar radius.

ECRB. The thumb adducts against the side of the index finger over a broad area when the wrist is extended. Arthrodesis of the IP joint causes the tenodesed FPL tendon to flex the metacarpophalangeal (MP) joint. Originally, the annular ligament (flexor pulley at the thumb MP joint) was divided to increase the mechanical advantage of FPL (Fig. 4), but a flexion deformity of the MP joint and excessive bowstringing of FPL led to the discontinuation of this release. 


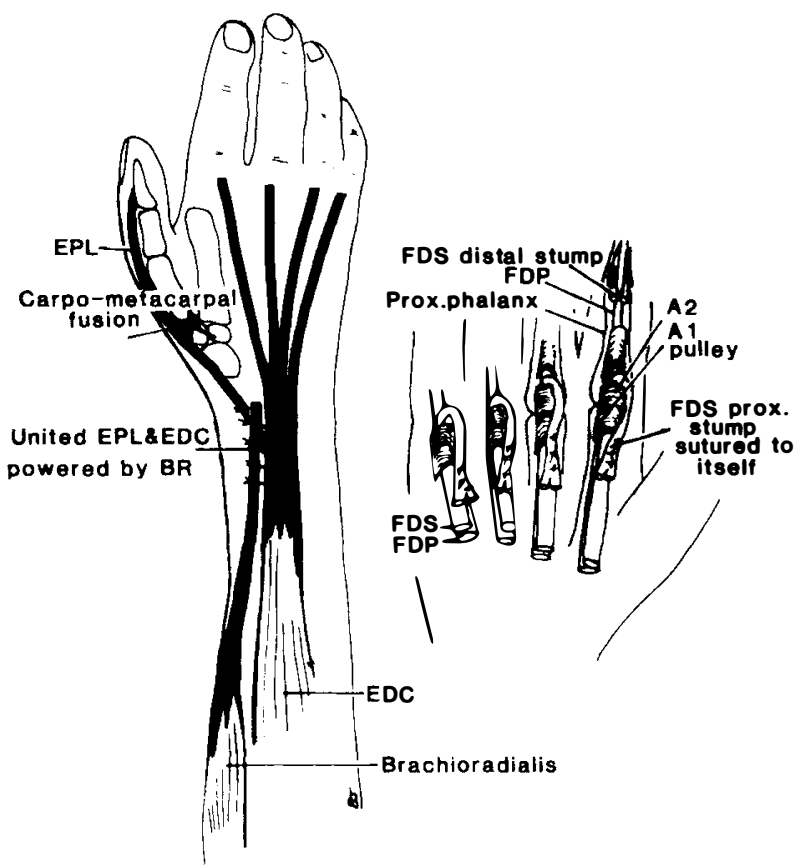

Figure 6 Zancolli's extensor phase: Carpo-metacarpal fusion in 45 degree abduction and 20 degrees extension, thumb (EPL) and finger (EDC) extensors powered by brachioradialis. 'Lasso' of flexor digitorum superficialis (FDS) tendons looped through fibrous flexor sheaths and sutured to themselves proximal to the Al pulleys.

Excessive metacarpophalangeal (MP) joint flexion may be limited by tenodesis of the extensor tendons to the metacarpal. If the wrist extensors do not need reinforcement, brachioradialis (BR) may be transferred to abductor pollicis longus or one of the thumb extensors to provide active opening of the key grip. Alternatively, extensor tendons may be tenodesed to the dorsal radius, usually as a secondary procedure. Brachioradialis is freed from its bony and fascial attachments proximally to achieve an excursion of 4 to $5 \mathrm{~cm}$. Recently, Moberg (McDowell et al., 1986) has adopted Brand's suggestion of passing FPL from the intact annular ligament, deep to the flexor tendons of the fingers to the ulnar side of the palm, then proximally through Guyon's canal to the site of tenodesis to the volar radius. One need not be concerned about damage to the ulnar nerve as it is not functioning in these patients. Adductor function is said to be improved (Fig. 5).

Hentz (1983) found that some patients had difficulty positioning the other fingers in preparation for lateral gripping, particularly if they lacked natural flexor tenodesis. A Zancolli 'lasso' procedure (Zancolli, 1979) or volar transfer of the paralysed extensor indicis (McDowell et al., 1986) may correct MP joint hyperextension.

Occasionally, as a secondary procedure, the FPL tenodesis needs tightening or the MP joint needs stablising by extensor tenodesis or arthrodesis. Extruded Kirschner wires need replacement. Threaded pins (Hiersche and Waters, 1985) or formal arthrodesis may be indicated. 


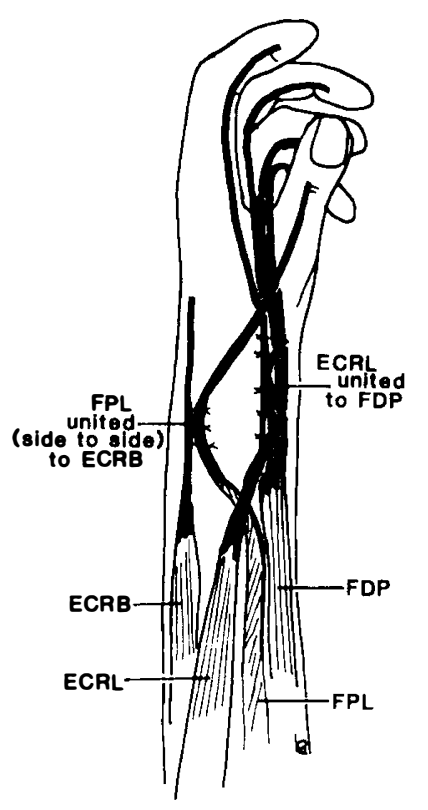

Figure 7 Zancolli's Flexor phase: Extensor carpiradialis longus (ECRL) woven into the flexor digitorum profundus (FDP) tendons to provide grasp. Pinch is provided by side to side suture of flexor pollicis longus (FPL) and extensor carpi radialis brevis (ECRB).

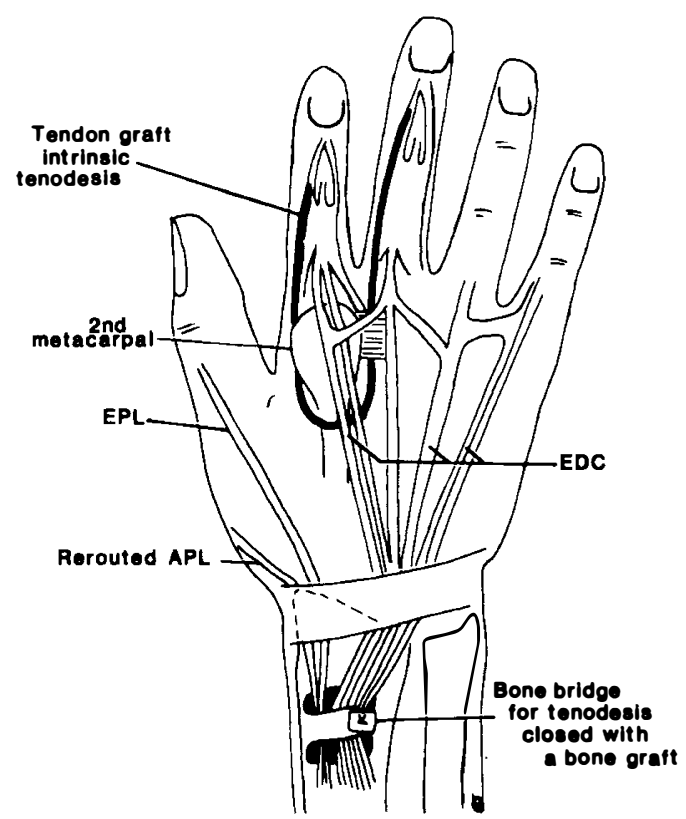

Figure 8 House's extensor phase: Extensor digitorum communis (EDC), extensor pollicis longus (EPL) and abductor pollicis longus (APL) are tenodesed to the dorsal radius (passed under a bridge of cortical bone completed with a graft). Intrinsic tenodesis of index and middle fingers is achieved by a loop of tendon graft passed through the lumbrical canals to the extensor expansions. 


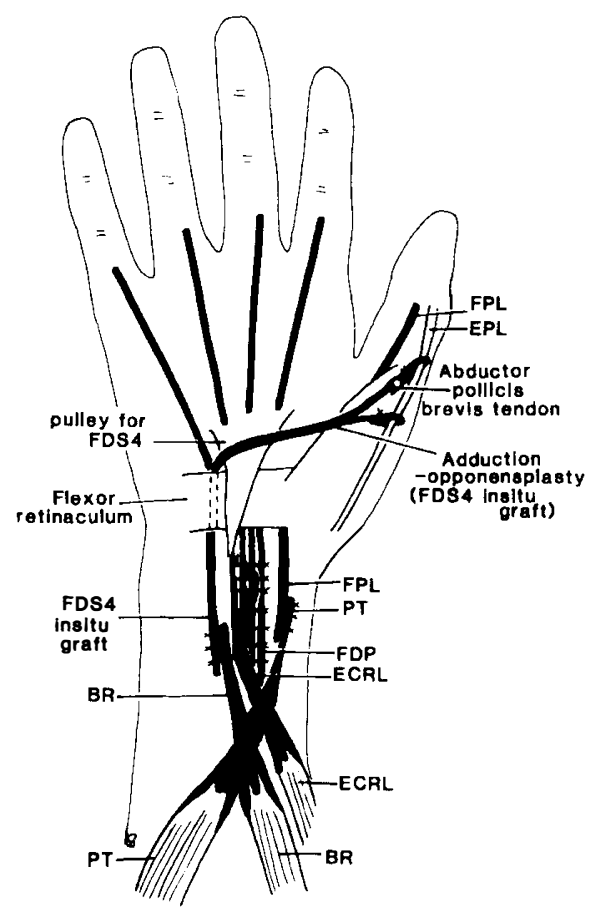

Figure 9 House's Flexor phase: Transfer of extensor carpi radialis longus (ECRL) into flexor digitorum profundus (FDP) and pronator teres (PT) into flexor pollicis longus (FPL).

Brachioradialis (BR) is transferred into an in situ graft of flexor digitorum superficialis to the ring finger (FDS4), and passes around the palmar aponeurosis which acts as a pulley, as an adductionopponensplasty.

\section{Zancolli pattern}

Zancolli (1975) performs a procedure consisting of an extensor phase and then a flexor phase for limbs classified as belonging to groups 3, 4 and 5 (Zancolli Group 2 B). In the past he also transferred supernumerary extensor carpi radialis muscles but he has found these muscles to be unreliable.

The extensor phase (Fig. 6) involves fusion of the thumb carpo-metacarpal (CMC) joint in a position described by Zancolli (1975 and 1979) as $45^{\circ}$ of antepulsion and $20^{\circ}$ to $30^{\circ}$ of radial abduction and transfer of the BR to finger and thumb extensor tendons. The MP joints of the fingers, which tend to hyperextend due to intrinsic paralysis, are stablised by the 'lasso procedure'. This consists of looping the flexor digitorum superficialis (FDS) tendons through the A2 pulleys of the fibrous flexor sheaths and suturing each tendon to itself with some tension just proximal to the Al pulley. In upper motor neuron paralysis, the superficial flexor maintains its muscle mass, its visco-elastic properties and its stretch reflex arcs. There is a tenodesis effect with wrist extension. In Group 4, pronator teres is transferred into flexor carpi radialis.

Four to 6 months later, the flexor phase (Fig. 7) is completed. It consists of transfer of ECRL into flexor digitorum profundus (FDP), to provide grasp. The FPL tendon may be activated by side to side suture to ECRB or by tenodesis to the volar radius. Activation by a supernumerary ECR is no longer 


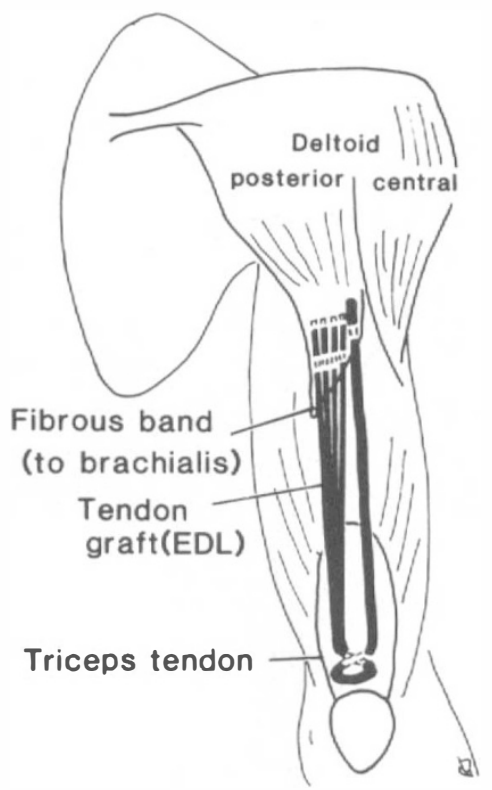

Figure 10 Transfer of the posterior deltoid to the triceps tendon using a free graft of toe extensor tendons (extensor digitorum longus EDL). A fibrous band from the deltoid to the brachialis reinforces the woven tendon graft.

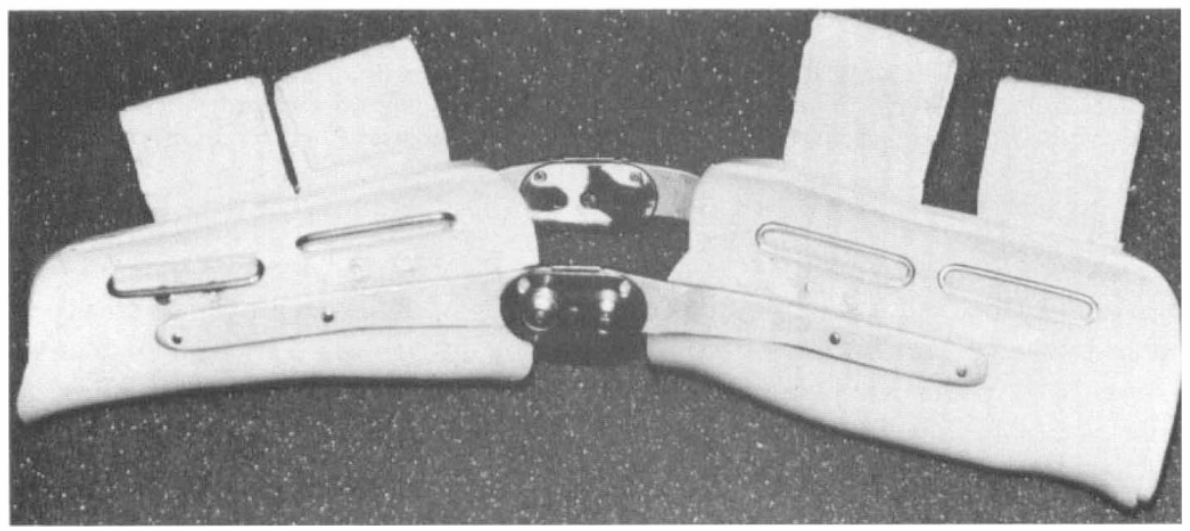

Figure 11 Adjustable hinge splint to limit elbow flexion during the mobilisation phase following deltoid to triceps transfer.

favoured. Accurate lateral pinch may be achieved and CMC joint fusion seems not to interfere with weight bearing functions.

For Group 1 limbs (Zancolli Group I B), the following procedures are performed: Deltoid to triceps transfer; thumb MP joint arthrodesis; tenodesis of FPL, EPL and abductor pollicis longus (APL). BR is transferred into ECRB providing active wrist extension. For Group 2 limbs, (Zancolli Group 2 A), the following are performed: thumb MP joint arthrodesis; tenodesis of EPL, APL and extensor digitorum communis (EDC). A FDS 'lasso' procedure is added and, at a second stage, BR is transferred into flexor digitorum profundus (FDP) to give active grasp. Pinch is provided by FPL tenodesis (Zancolli, 1979). 


\section{House pattern}

For Groups 2 and 3, House (1985) fuses the CMC joints of the thumb, performs an EPL tenodesis and either a FPL tenodesis (Group 2) or transfer (Group 3). For Group 4 limbs, House et al. (1976) recommended an extensor phase consisting of tenodesis of EDC, EPL and APL to the radius. An 'intrinsic balance tenodesis' is created by a free tendon graft fixed to the extensor apparatus of the index and middle fingers. It is routed through the lumbrical canals (volar to the deep transverse metacarpal ligament) to be fixed to the dorsal surface of the metacarpal of the index finger (Fig. 8). In the flexor phase (Fig. 9), ECRL is transferred into FDP. FPL and an adduction-opponensplasty are powered by PT and BR. The adduction-opponensplasty consists of an in situ graft using the tendon of the FDS to the ring finger. This is passed from a pulley created by the edge of the palmar aponeurosis, just distal to the flexor retinaculum, and then tunnelled subcutaneously across the palm to insertions into abductor pollicis brevis and extensor pollicis longus, both distal to and proximal to the MP joint. Techniques of pressure lifts and transferring, which involve weight bearing on the proximal palm, result in forceful extension of both the wrist and the thumb. We believe that this is contraindicated following an opponensplasty due to the risk of rupture, but patients may transfer by resting on the clenched fist.

\section{Other patterns}

Freehafer (1984) has constructed an opponensplasty and powered FDP with one muscle only. Brachioradialis has been transferred into FPL with thumb IP joint pinning and an antagonistic deltoid to triceps transfer. BR has powered an opponensplasty and ECRL has powered FDP in Group 3 limbs (Freehafer Group III) (Freehafer et al., 1974). In Group 4 limbs, pronator teres usually powers the opponensplasty and BR is inserted into FDP (Freehafer et al., 1984). Extensor tenodeses were performed secondarily if natural tenodesis was insufficient to prevent a flexion deformity of the fingers.

Waters et al. (1985) reported transfer of BR into FPL in Group 2 or better limbs. The thumb IP joint was fixed with a specially designed Moberg screw (threaded pin) and thumb extensor tendons were tenodesed to the dorsum of the metacarpal. The pinch pressure, which averaged nearly $2 \mathrm{~kg}$, was proportional to the wrist extension torque, but it was also related to the strength of the triceps. An active pinch is relatively independent of wrist position and is more precise than a FPL tenodesis. If the wrist extensor torque is less than 10 feetpounds, BR is best transferred to reinforce ECRB and FPL is tenodesed.

Lamb (1983) reports transfer of ECRL into FDP and BR into FPL in 45 hands. In 5 additional hands, an ECRL to FDP transfer was combined with a Moberg type FPL tenodesis with good results. 16 limbs had posterior deltoid to triceps transfers.

The problems of C6 and C7 quadriplegia have been approached differently by Kiwerski (1982). He divided the musculocutaneous nerve, usually between branches given off to the long and short heads of biceps and above the branch to the brachialis and anastomosed it to the median nerve. Of 20 patients, 9 were 


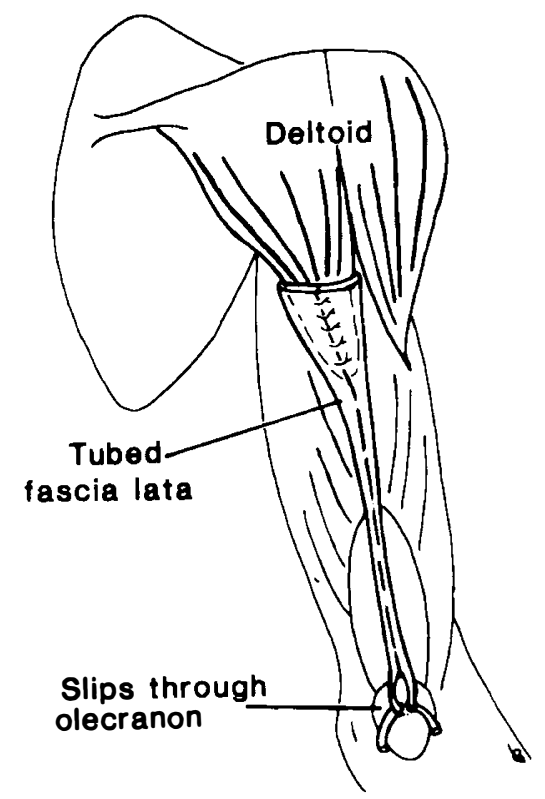

Figure 12 Fascia lata has been tubed about the posterior deltoid and is inserted into the olecranon via a drill hole.

reported as having good results with grip and some sensory recovery, 6 were reported to have a weaker but useful grip with poor sensory recovery and 5 had poor results.

\section{Elbow extension procedures}

Reasonably strong active elbow extension may be restored by transfer of the posterior part of the deltoid muscle $(\mathrm{C} 5,6$, axillary nerve innervation) to the aponeurosis of the triceps muscle ( $C 7,8$ radial nerve innervation), using free extensor tendon grafts from the leg (Moberg, 1975) (Fig. 10). As contraction of the posterior part of the deltoid muscle is synergistic with elbow extension, reeducation of the transfer is relatively simple. The transferred muscle is blocked with a local anaesthetic to prevent unprotected contraction during recovery from general anaesthesia. The elbow is splinted in extension for 6 weeks followed by mobilisation restricted to $10^{\circ}$ additional flexion per week (Fig. 11). Shoulder movement is also restricted. Any loss of active extension, indicating some stretching or dehiscence is treated by immobilisation in extension for 1 to 2 weeks (Moberg, 1978). Permanent loss of full active extension after this procedure may be treated by distal advancement of the osteotomised triceps insertion on the olecranon (McDowell et al., 1986).

De Benedetti (1979) found that, in 14 posterior deltoid transfers, the average isotonic extensor power was $1.7 \mathrm{~kg}$ with a range of 0.5 to $4.1 \mathrm{~kg}$. The requirement for prolonged splinting and controlled mobilisation has led a number of authors to suggest alternative procedures.

Hentz et al. (1983) recommended wrapping a wide strip of tubed fascia lata 


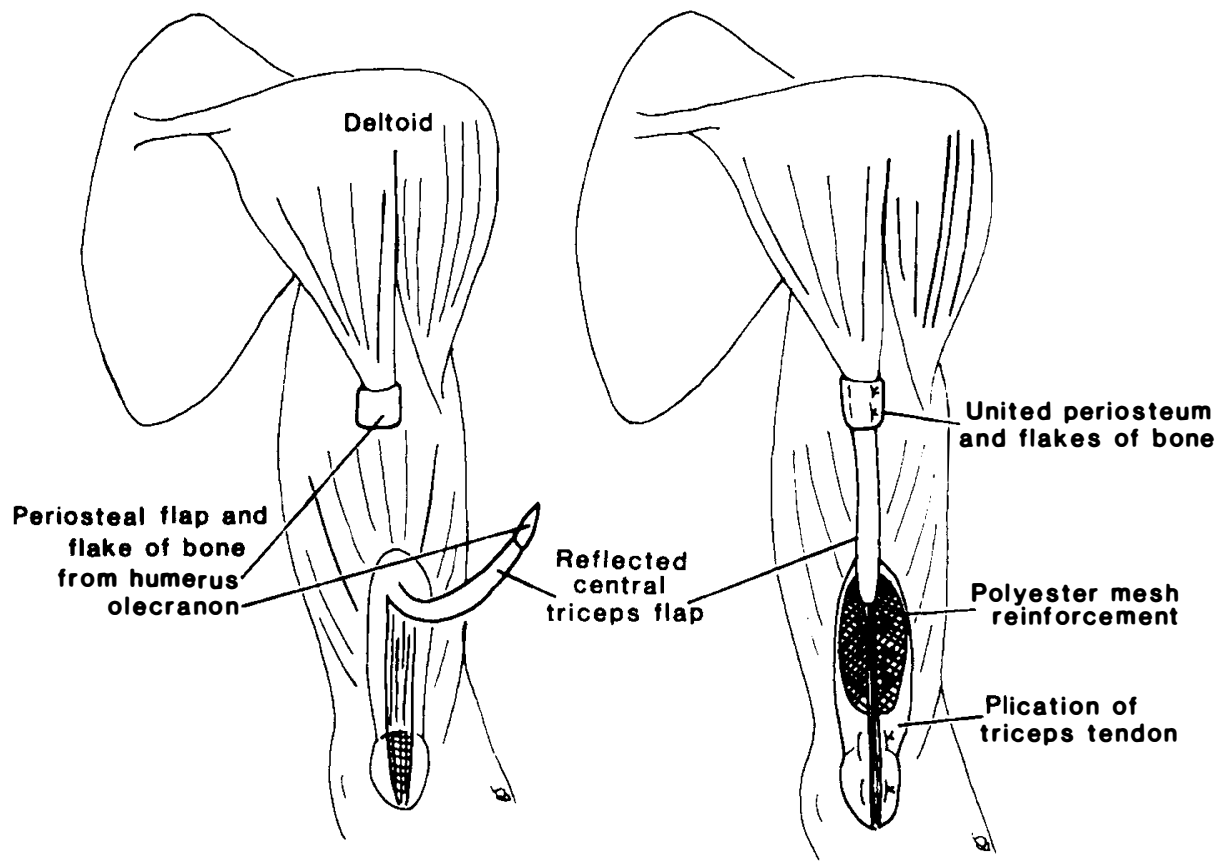

Figure 13 A flap of periosteum and attached flakes of bone have been raised in continuity with the posterior deltoid insertion and the central portion of the triceps tendon. The central triceps tendon is reflected proximally to be wired to the posterior deltoid. The triceps tendon is plicated and the point of reflection is reinforced with polyester mesh.

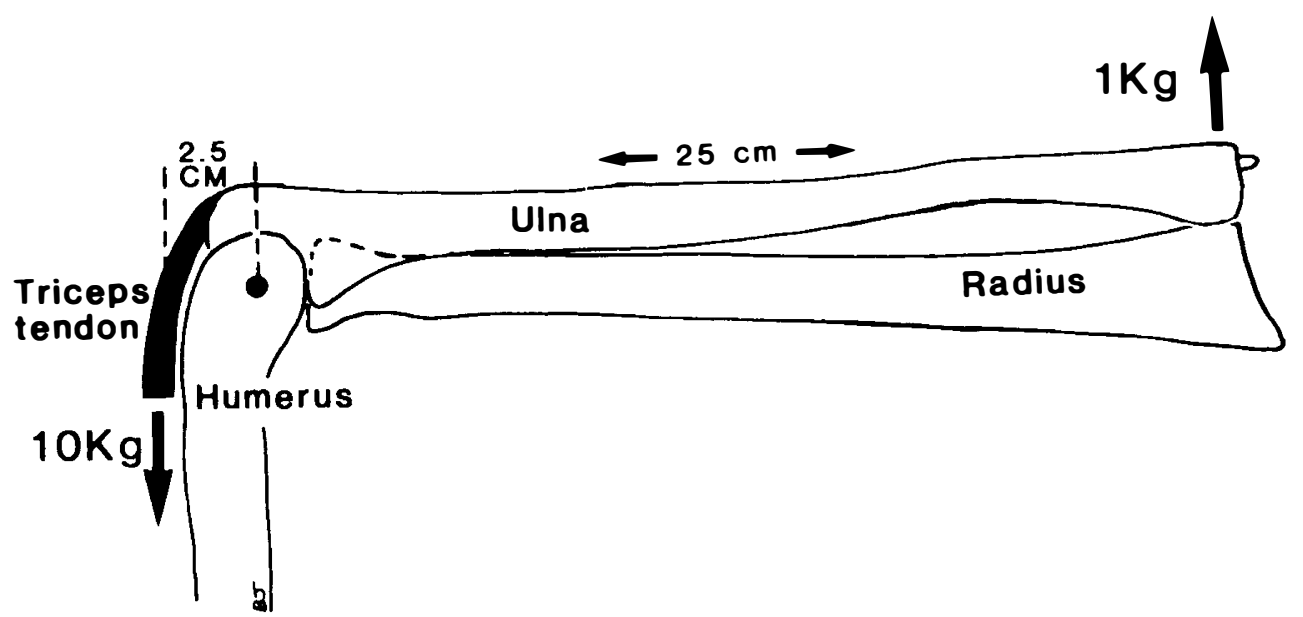

Figure 14 The ratio of the lengths of the ulna proximal to and distal to the fulcrum of the trochlear notch is approximately $1: 10$. A tension of $10 \mathrm{~kg}$ in the triceps tendon will produce a force of $1 \mathrm{~kg}$ at the distal end of the ulna.

around the distal $4 \mathrm{~cm}$ of the detached posterior part of the deltoid muscle. The distal end of the fascia is passed through holes drilled in the olecranon (Fig. 12). After $4 \frac{1}{2}$ weeks immobilisation, $30^{\circ}$ of flexion is permitted with $15^{\circ}$ further flexion per week. Hentz noted that, without active elbow extension, a flexion contracture 


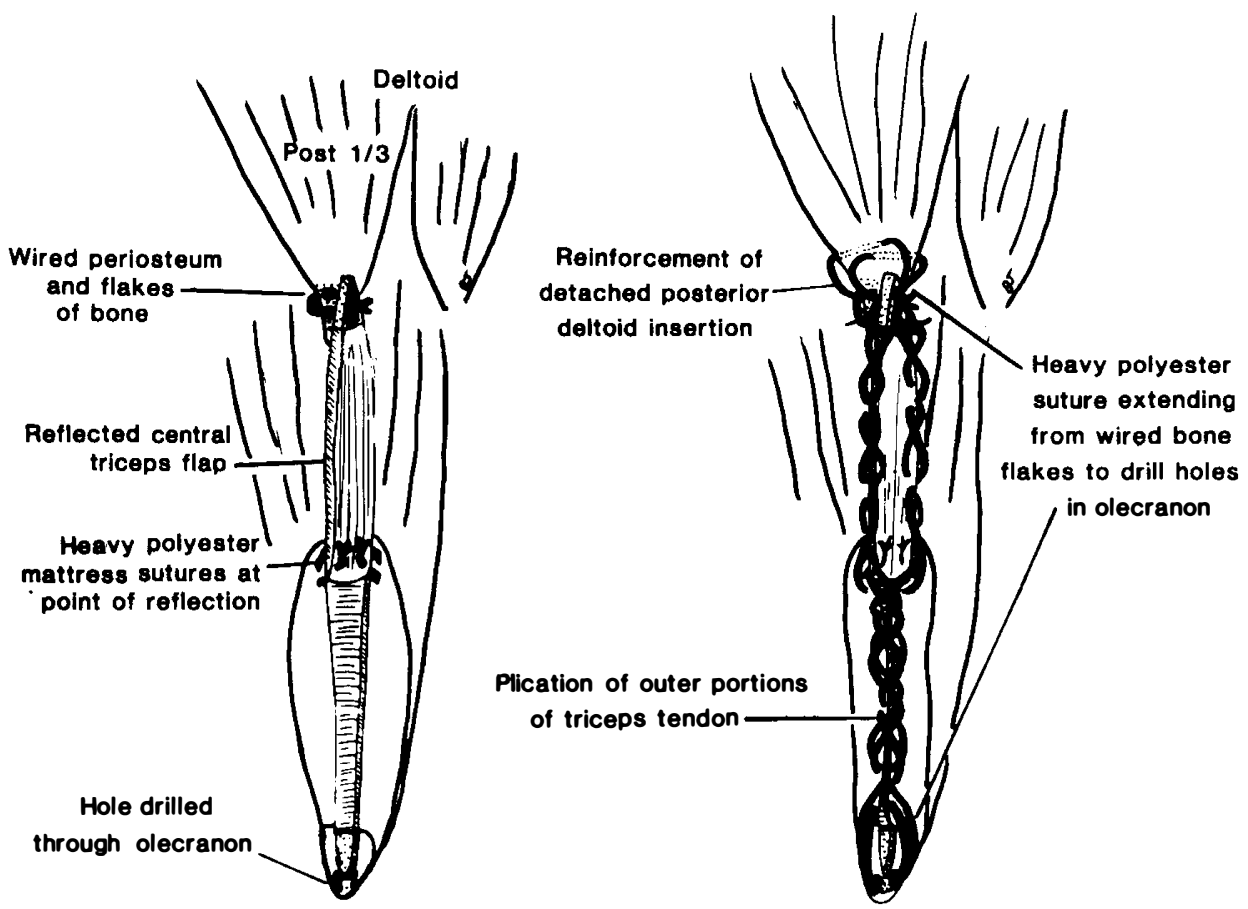

Figure 15 The central strip of the triceps tendon and part of the bone to which it is inserted is reflected proximally to be wired to detached bone at the insertion of the posterior third of the deltoid muscle. The point of reflection is reinforced by strong mattress sutures of No. 1 polyester (which has a knot pull strength of about $5 \mathrm{~kg}$ ). Heavy (No. 1) polyester sutures are also threaded through a drill hole in the olecranon and then passed as plicating stitches along the inner margins of the lateral strips of triceps tendon to the site of reflection of the central strip of the tendon. The sutures then anchor the margins of the poorly vascularised central strip of tendon to the vascular triceps muscle and are finally secured to the wire suture approximating the detached fragments of bone. These polyester sutures take the strain of the deltoid to triceps transfer and thus allow early mobilisation while the tendon transfer itself heals and develops strength.

of the elbow may develop as a distally transferred brachioradialis gains strength. This causes secondary loss of wrist flexion as the brachioradialis shortens. Freehafer (1984) used tibialis anterior tendon as a free graft and immobilised the elbow in $20^{\circ}$ of flexion for 4 weeks before commencing active exercise. Castro-Sierra and Lopez-Pita (1983) reflected a central flap of triceps tendon proximally with a portion of periosteum. This was fixed to the posterior part of the deltoid muscle and its detached insertion. This procedure has been modified by the authors of this paper to include flakes of bone which may be securely wired together (Fig. 13). Polyester mesh was used to reinforce the reflected triceps tendon flap. Castro-Sierra and Lopez-Pita claimed to reduce the immobilisation time to a total of 35 days with satisfactory results in 10 cases, but despite reinforcement, the triceps tendon flap is prone to tear up at the point of reflection (Johnstone et al., 1987).

Zancolli (1979) transfers the biceps tendon to the triceps insertion if a musculocutaneous nerve block demonstrates supinator function. The power of elbow flexion is reduced by $24^{\circ}{ }_{0}$ (McDowell et al., 1986). In six cases elbow extension power ranged from 0.25 to $0.9 \mathrm{~kg}$ (Zancolli, 1979). 
As the ulna proximal to and distal to the fulcrum of the trochlear notch has a length ratio of about 1:10 (Figure 14), $10 \mathrm{~kg}$ (98 Newton) of tension is present at the triceps insertion, to balance a force of $1 \mathrm{~kg}$ applied to the ulnar styloid (approximately the force required to extend the forearm against gravity). As the posterior deltoid to triceps transfer may develop a maximum extension force of over $6 \mathrm{~kg}$ at the ulnar styloid, (Johnstone et al., 1987), a tension of 50 to $60 \mathrm{~kg}$ will be reached in the posterior deltoid-triceps complex. Tendon junctions weaken before gaining strength during the fibroplastic and later remodelling phases of healing and may show patchy central necrosis. Therefore any programme of early mobilisation must consider these potentially adverse changes as well as the initial strength of the posterior deltoid-triceps complex.

Freehafer and co-workers (Lacey et al., 1986) have documented the length tension characteristics of the posterior deltoid muscle using intra-operative electrical stimulation. After adequate dissection, the mean total excursion was $7.31 \pm 1.23 \mathrm{~cm}$. This is in excess of the $3 \mathrm{~cm}$ triceps tendon excursion, but the deltoid-triceps complex must be active over a range of combined shoulder and elbow positions. The length-tension curves were rather flat however maximum active force was developed when the muscles were stretched to the region of the reference point at the humeral insertion with the arm by the side. To gain maximum benefit from this transfer over a range of shoulder and elbow positions, Freehafer recommends stretching the posterior deltoid level with its original insertion and fixing the tension by attaching the distally inserted tendon graft with the elbow at $90^{\circ}$ and the arm by the side. This is a departure from established practice and does not allow for stretching of the graft or for a degree of junctional dehiscence. Transfers performed with the muscle stretched to its limits, the shoulder abducted and the elbow extended must elongate at some point to permit controlled elbow flexion. This elongation may be monitored radiologically if metal clips are placed at known intervals along the deltoid-grafttriceps complex. It is now our practice to reinforce a proximally reflected flap of central triceps tendon with lengths of heavy polyester suture passed between the detached posterior deltoid insertion and drill holes through the olecranon. Tearing at the point of reflection of the triceps tendon flap (really an aponeurosis) is avoided as the polyester takes much of the strain during early mobilization. The triceps tendon flap which initially has limited strength, probably acts as a connective tissue graft providing fibroplastic tissue which forms scar or even 'neotendon' about the polyester suture framework. (Figure 15)

Procedures used in $\mathrm{C} 7$ quadriplegia: International classification groups 5,6 and 7

\section{Group 5) Flexor Carpi Radialis (FCR) Functioning \\ Group 6) Finger Extensors Functioning \\ Group 7) Thumb Extensors Functioning}

House and Shannon (1985) have compared the results of different procedures for each hand of six patients, whose limbs were classified as belonging to Groups $\mathrm{Cu}$ 4, 5, 6 and 7. Eight of these limbs belonged to Group $\mathrm{Cu}$ 5. Method I, which has already been detailed (House 1985), can be summarised as:

Extensor phase: EDC, EPL and APL tenodesis and an intrinsic balance (Fig. 8) tenodesis using a free tendon graft. 


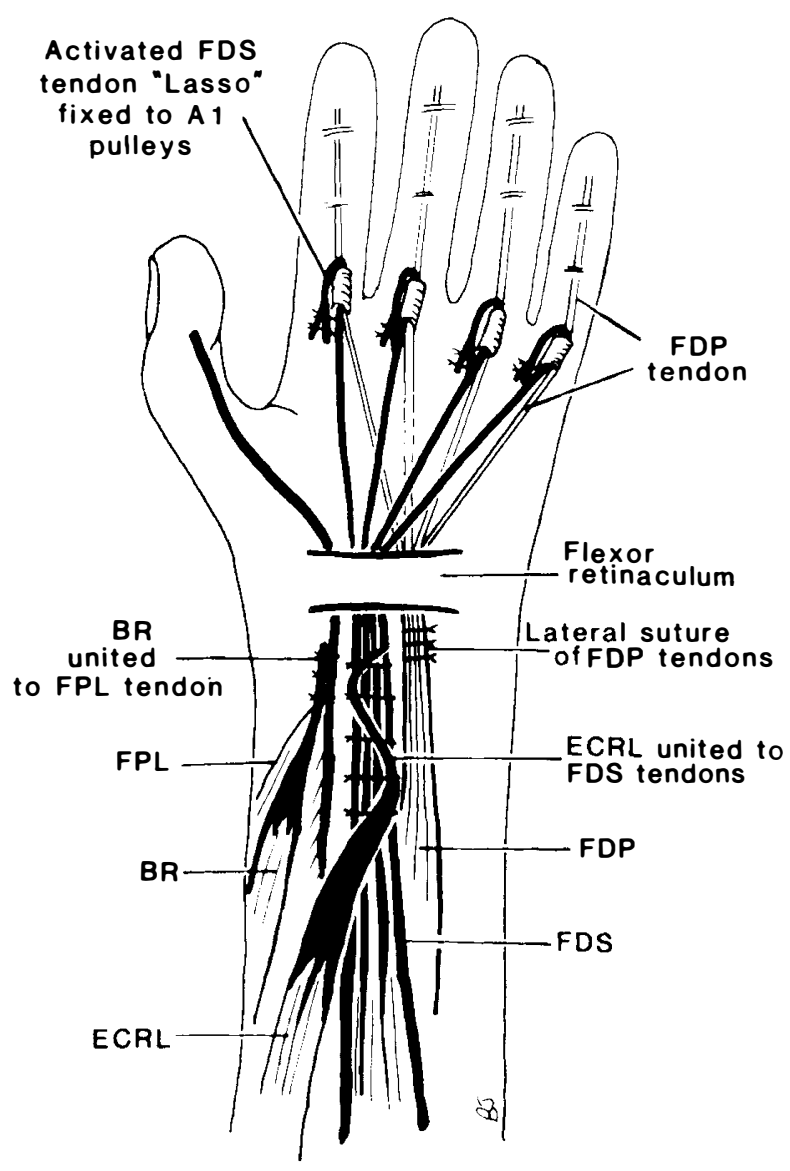

Figure 16 Zancolli's treatment of group 8 hands (partial finger flexion and intrinsic paralysis), (Stage I): Transfer of brachioradialis (BR) to flexor pollicis longus (FPL), flexor digitorum superficialis (FDS) 'lasso' activated by extensor carpi radialis longus (ECRL) and suture of the flexor profundus tendon of the index finger (FDP) to the other profundus tendons.

Flexor phase: ECRL transferred to FDP, PT to FPL, BR to ring finger FDS (Fig. 9) adduction-opponensplasty.

Method II is a modification of the previously detailed Zancolli pattern (Figs 6 and 7):

Extensor phase: Thumb CMC joint fusion BR transferred to EDC and EPL. Flexor phase: FDS 'lasso' to provide intrinsic balance (previously an extensor phase procedure) ECRL to FDP

PT to FPL (as FCR functions, PT is not required to power wrist flexion).

Pronator teres is extended by including a 5 to $7 \mathrm{~cm}$ strip of periosteum distal to the radial insertion to reach the FPL tendon. $\mathrm{Cu} 7$ limbs require only the flexor phase. Method I was used on the right in three patients and Method II used on the right in the other three. Method II was used on the side with less 


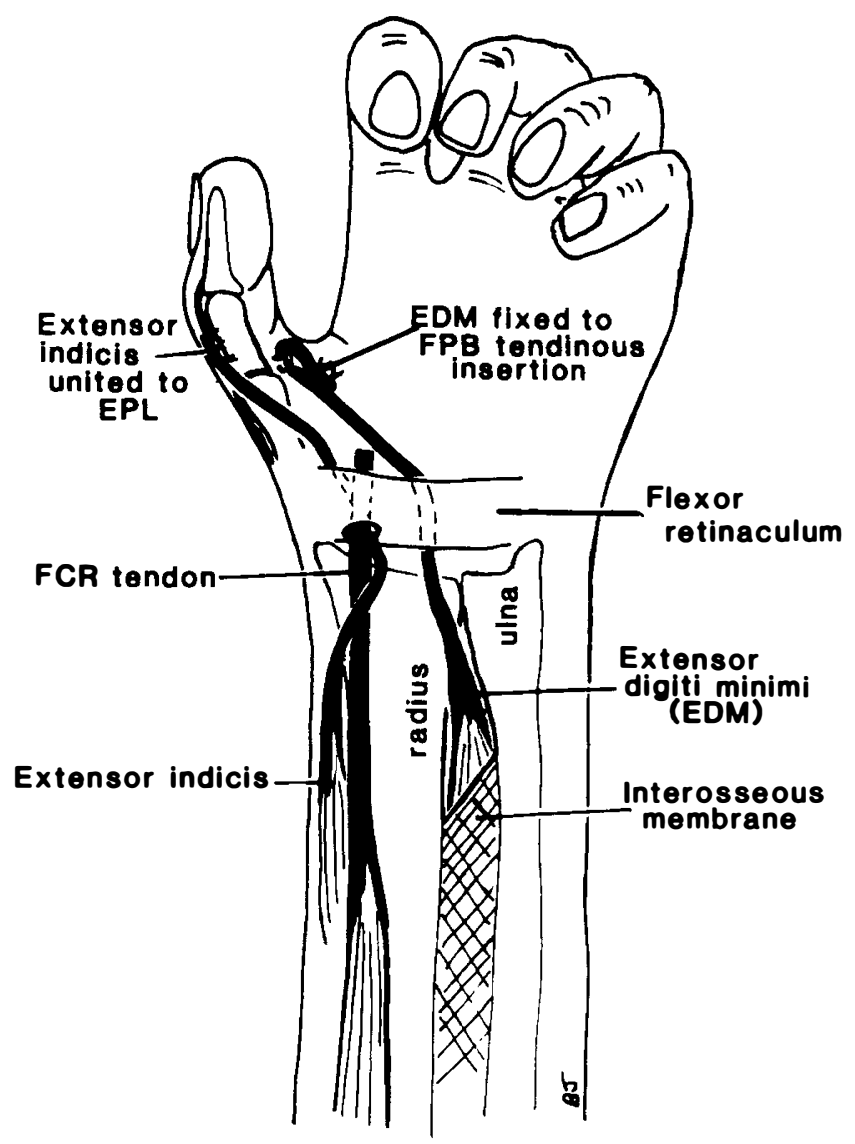

Figure 17 Zancolli's treatment of group 8 hands (Stage II): Extensor indicis is re-routed about the tendon of flexor carpi radialis (FCR) to insert into extensor pollicis longus (EPL). Extensor digiti minimi (EDM) is passed through the interosseous membrane and carpal tunnel to the tendinous insertion of flexor pollicis brevis (FPB).

residual function in three patients with asymmetric functional levels. Limbs in Group $\mathrm{Cu} 6$ and $\mathrm{Cu} 7$ had the best results. The average pinch and grip measurements were 3.73 and $6.86 \mathrm{~kg}$ for Method I and 3.31 and $7.42 \mathrm{~kg}$ for Method II respectively. Patients did not prefer one hand, but found that the difference resulted in specialisation of each hand for certain activities, which was especially valuable for two handed activities. Method I hands were preferred for manipulating large objects, while Method II hands were preferred for handling smaller items because of the stable but less mobile thumb.

Feeehafer (1984) reports an opponensplasty similar to that of House, except that the tendon is inserted into the abductor pollicis brevis and is powered by $\mathrm{BR}$ or PT, (FCR, ECRL and palmaris longus have also been used). EPB has been rerouted subcutaneously across the palm and attached to ECRL which had been brought around the ulnar side of the forearm. FDP was usually powered by BR, however PT, FCR and ECRL have been used to provide strong effective grasp. 


\title{
Procedures used in C8 quadriplegia: International classification groups 8 and 9
}

\author{
Group 8) Partial Finger Flexor Function \\ Group 9) Full Finger Flexors. Only Intrinsics Lacking
}

House (1985) uses a Zancolli FDS 'lasso' to provide intrinsic balance and an adduction-opponensplasty. Flexor or extensor carpi ulnaris may be used as a motor. Abductor pollicis brevis (APB) function is restored by dividing EPB at its insertion and re-routing it around the palmaris longus tendon at the wrist and inserting it into the APB tendon. This can restore true opposition and a three point pinch.

Zancolli (1979) treats Group 8 (Zancolli Group 4A) hands in two stages.

Stage I BR into FPL.

(Fig. 16) Side to side suture of FDP tendon of the index finger to the others. Active indirect 'lasso' by transferring ECRL into FDS.

Stage II Extensor indicis is re-routed around the FCR tendon and through its (Fig. 17) tunnel to EPL to restore APB function.

Extensor digiti minimi is passed through the inter-osseous membrane, through the carpal tunnel and inserted into the tendinous insertion of flexor pollicis brevis of the thumb to stabilise the metacarpophalangeal joint and provide adduction.

In Group 9 (Zancolli Group 4 B II), the FDS 'lasso’ does not need activation.

\section{Acknowledgements}

The authors wish to thank the following for assistance to $\mathrm{Mr}$ J. A. Buntine during a period of sabbatical leave: A. A. Freehafer, J. H. House, C. Hamlin, R. L. Waters. We are indebted to Professor Erik Moberg for his inspirational contributions to international meetings and literature which have provided guidance and motivation for our work. Mrs Robyn Tolley and Mrs Hazel Mapperson of the Department of Surgery, Austin Hospital, kindly typed numerous drafts of this paper.

\section{References}

Bunnell S 1948 Surgery of the Hand, 2nd Ed. Philadelphia. J.B. Lippincott Company.

Castro-Sierra A, Lopez-Pita A 1983 A New Surgical Technique to Correct Triceps Paralysis. Hand 15:42-46.

De Benedetti M 1979 Restoration of Elbow Extension Power in the Tetraplegic Patient using the Moberg Technique. Fournal of Hand Surgery 4:86-89.

Freehafer AA, Von HaAm E, Allen V 1974 Tendon Transfers for Improved Grasp after Injuries of the Cervical Spinal Cord. Fournal of Bone and foint Surgery 56A:951-959.

Freehafer AA, Peckham PH, Keith MW 1979 Determination of Muscle-Tendon Unit Properties during Tendon Transfer. Fournal of Hand Surgery 4:331-339.

Freehafer AA, Kelly CM, Peckham PH 1984 Tendon Transfer for the Restoration of Upper Limb Function after a Cervical Spinal Cord Injury. Fournal of Hand Surgery 9A:887-893.

HentZ VR, BRown M, KeOShian LA 1983 Upper Limb Reconstruction in Quadriplegia: Functional Assessment and Proposed Treatment Modifications. Fournal of Hand Surgery 8:119-131.

HIERSCHE DL, WATERS R L 1985 Interphalangeal Fixation of the Thumb in Moberg's Key Grip Procedure. Fournal of Hand Surgery 10A:30-32.

House JH, GWATHMEY FW, LuNDSGAARD DK 1976 Restoration of Strong Grasp and Lateral Pinch in Tetraplegia due to Cervical Spinal Cord Injury. Fournal of Hand Surgery 1:152-159.

HousE JH 1985 Reconstruction of the Thumb in Tetraplegia Following Spinal Cord Injury. Clinical Orthopaedics 195:117-128. 
House JH, Shannon MA 1985 Restoration of Strong Grasp and Lateral Pinch in Tetraplegia. A Comparison of Two Methods of Thumb Control in Each Patient. Fournal of Hand Surgery 10A:21-29.

Johnstone BR, Buntine JA, Slattery PG, Jordan CJ, Philip KM 1987 Surgical Rehabilitation of the Upper Limb in Quadriplegia. Australian and New Zealand fournal of Surgery 57:917926.

KIWERSKI J 1982 Recovery of Simple Hand Function in Tetraplegia Patients Following Transfer of the Musculocutaneous Nerve into the Median nerve. Paraplegia 20:242-247.

Lacey SH, Wilber RG, Peckham PH, Freehafer A A 1986 The Posterior Deltoid to Triceps Transfer: A Clinical and Biomechanical Assessment. Fournal of Hand Surgery 11A:542-547.

LAMB DW, ChAN KM 1983 Surgical Reconstruction of the Upper limb in Traumatic Quadriplegia. Fournal of Bone Foint Surgery 65B:291-298.

MCDowell CL, Moberg EA, House JH 1986 The Second International Conference on Surgical Rehabilitation of the Upper Limb in Tetraplegia (Quadriplegia). Fournal of Hand Surgery 11A:604-608.

Moberg E 1975 Surgical Treatment for Absent Single-hand Grip and Elbow Extension in Quadriplegia. Fournal of Bone and foint Surgery 57 A:196-206.

Moberg E 1978 The Upper Limb in Tetraplegia: A New Approach to Surgical Rehabilitation. Stuttgart George Thieme Publishers.

Waters RL, MoORe KR, Graboff SR, Paris K 1985 Brachioradialis to Flexor Pollicis Longus Tendon Transfer for Active Lateral Pinch in the Tetraplegia. Fournal of Hand Surgery 10A:385-391.

ZaNCOLl E 1975 Surgery for the Quadriplegic Hand with Active, Strong Wrist Extension Preserved. A Study of 97 Cases. Clinical Orthopaedics 112:101-113.

ZanColli E 1979 Structural and Dynamic Basis of Hand Surgery. 2nd Ed. Philadelphia. J.B. Lippincott Company. 